\title{
Clinicopathological characteristics and prognostic analysis of lymphoepithelial carcinoma of salivary gland: a population-based study
}

\author{
Jia-Qi Wang", Rong-Xin Deng", Hui Liu", Yuan Luo, Zhi-Cheng Yang \\ Department of Oral and Maxillofacial Surgery, Shanghai Stomatological Hospital, Fudan University, Shanghai, China \\ Contributions: (I) Conception and design: Y Luo, ZC Yang; (II) Administrative support: ZC Yang; (III) Provision of study materials or patients: JQ \\ Wang, ZC Yang; (IV) Collection and assembly of data: JQ Wang, H Liu; (V) Data analysis and interpretation: JQ Wang, RX Deng; (VI) Manuscript \\ writing: All authors; (VII) Final approval of manuscript: All authors. \\ "These authors contributed equally to this work. \\ Correspondence to: Zhi-Cheng Yang. Department of Oral and Maxillofacial Surgery, Shanghai Stomatological Hospital, Fudan University, No. 356 \\ East Beijing Road, Shanghai 200001, China. Email: zhichengyang1998@sina.com.
}

\begin{abstract}
Background The purpose of this study was to elucidate the clinicopathological characteristics of patients with lymphoepithelial carcinoma of salivary gland (LEC-SG) and determine the factors associated with survival.

Methods: A total of 179 LEC-SG patients from the Surveillance, Epidemiology, and End Results (SEER) database between 1975 to 2016 were included. The clinicopathological characteristics and overall survival of LEC-SG patients were described, and the features affecting prognosis were further determined using Kaplan-Meier Curves and Cox survival analysis.

Results: The median overall survival of LEC-SG patients was 206 months, and the 1-, 5-, 10- and 20-year survival rates were $91.0 \%, 80.2 \%, 66.4 \%$, and $37.6 \%$, respectively. The prognoses were significantly associated with age, ethnicity, marital status, tumor invasion, as well as lymph node metastases $[\mathrm{P}<0.01$ for all). Surgical resection could significantly improve the prognosis of this disease (median overall survival (mOS): 219 vs. 68 months, $\mathrm{P}<0.01$ ]. Postoperative radiotherapy could improve long-term survival and decrease the risk of death among patients who survive exceed 10-year after surgery. The Cox regression analysis showed that old age ( $>60$ years) and lymph node metastases were independently associated with poor survival $(\mathrm{P}<0.05$ for both). Conversely, the use of surgery was an independent favorable prognostic factor [hazard ratio (HR) 0.29, 95\% CI: 0.13-0.66].
\end{abstract}

Conclusions: LEC-SG patients had a favorable prognosis with a mOS of 206 months. Old age, lymph node metastases, the use of surgery were independently associated with survival of LEC-SG patients.

Keywords: Salivary gland tumor; lymphoepithelial carcinoma (LEC); Surveillance, Epidemiology, and End Results database (SEER database); prognosis

Submitted Apr 21, 2020. Accepted for publication Sep 03, 2020.

doi: $10.21037 / \mathrm{gs}-20-464$

View this article at: http://dx.doi.org/10.21037/gs-20-464

\section{Introduction}

Lymphoepithelial carcinoma (LEC) is a rare malignancy characterized by a poorly differentiated carcinoma accompanied by a prominent non-neoplastic lymphoplasmacytic infiltration (1). LEC was often occurred in the nasopharynx, accounting for $40 \%$ of all nasopharynx neoplasms (2). Except for the nasopharynx, it could also occur in other site in the head and neck region and previous studies showed LEC represents approximately $5 \%$ of head and neck cancers $(3,4)$. Epstein-Barr virus (EBV) infection 
is considered to be contributing factor for the initiation and development of this disease, so LEC has a unique ethnic predilection for Eskimo, Chinese and Japanese population $(5,6)$. In addition, LEC is also associated with tobacco and alcohol use (7). Similar to other pathological type, the combination of surgery and postoperative radiotherapy is the mainstay treatment paradigm for LEC patients (8). Due to significant lymphocytic infiltration in LEC tissue which could induce a strong anti-tumor immune response, the previous studies reported that LEC patients often had good prognosis $(8,9)$.

LEC of salivary gland (LEC-SG) is exceedingly rare and only comprises $0.4 \%$ of salivary malignancies (10). Due to its rarity, most knowledge and information about this disease comes from case report or case series. The clinicopathological characteristics and prognosis of LECSG patients has not fully defined. To better understand this rare malignancy, we conducted a population-based study to present the clinicopathological characteristics, and further determine the prognostic factors using the data of LEC-SG patients registered in Surveillance, Epidemiology, and End Results (SEER) database between 1975 to 2016. We present the following article in accordance with the STROBES reporting checklist (available at http://dx.doi.org/10.21037/ gs-20-464).

\section{Methods}

\section{Data source and participants}

The database 'SEER 18 Regs Custom Data with additional treatment fields, Nov 2018 Sub (1975-2016)' was searched. We accessed the SEER database with the dedicated software SEER*Stat (http://seer.cancer.gov/seerstat) and obtained all available cases of primary LEC located in salivary glands, with ICD-O-3 histology coding (ICD-O-3:8310/3) between 1975 and 2016. In each case, age at diagnosis, sex, ethnicity, TNM-AJCC stage, SEER historic stage, the use of radiotherapy/chemotherapy/surgery were recorded. The overall survival time was also isolated from the SEER database. The study was conducted in accordance with the Declaration of Helsinki (as revised in 2013).

\section{Statistical analysis}

The clinicopathological characteristics were summarized using counts and percentages. Student's $t$-tests and chisquare tests were used to evaluate the difference in continuous data and categorical data, respectively. The Kaplan-Meier Curves were drawn, survival analysis was assessed by Log-Rank test. The association of each characteristic with prognosis was evaluated using univariate and multivariate Cox regression models. MedCalc statistical software was used for all statistical analysis (Mariakerke, Belgium). A P value less than 0.05 was considered statistically significant.

\section{Results}

\section{Clinicopathological characteristics of LEC-SG patients}

Totally, 179 patients diagnosed with LEC-SG were identified from SEER database between 1975 and 2016 (Table 1). A total of 93 out of 179 patients were female and another 86 cases were male. The average age at initial diagnosis was 60.2 years $(60.2 \pm 18.2 \mathrm{yr})$. This disease was common in the parotid gland $(153 / 179)$, followed by submandibular gland (23/179). The distribution of the presenting stages of LEC of salivary gland was (63/179) distant stage, (77/179) regional stage, (20/179) localized stage. As for treatment regimen, most patients (166/179) underwent surgery, 119 patients (119/179) received radiotherapy after surgery and 33 patients received chemotherapy.

\section{Overall survival of LEC-SG patients}

As shown in Figure 1A, the median OS (mOS) of all 179 LECSG patients was 206 months (95\% CI: 143-262 months), with the 1-, 5-, 10- and 20-year survival rates of 91.0\%, $80.2 \%, 66.4 \%$, and $37.6 \%$, respectively. Patients with distant stage or regional stage (SEER historic stage) had relatively shorter overall survival time than those with localized stage, but no significantly statistical difference could be observed $(\mathrm{P}=0.12$, Figure $1 B)$. According to TNMAJCC stage classification, patients with advanced stage (III/ IV) had significantly shorter overall survival time than those with early stage (I/II) $(\mathrm{P}<0.05$, Figure 1C).

\section{Features influencing overall survival}

The stratification analysis showed that LEC-SG patients who were non-white/black ethnicity had significantly better prognosis than white/black individuals $(\mathrm{P}<0.05$, Figure $2 A)$. The prognoses of LEC-SG patients became much worse with increasing age, increasing tumor invasion and lymph 
Table 1 Clinicopathological characteristics of 179 patients with LEC of salivary gland

\begin{tabular}{|c|c|}
\hline Characteristics & Total \\
\hline Age (year) & $60.2 \pm 18.2$ \\
\hline \multicolumn{2}{|l|}{ Gender } \\
\hline Female & 93 (52.0\%) \\
\hline Male & $86(48.0 \%)$ \\
\hline \multicolumn{2}{|l|}{ Ethnicity } \\
\hline White & $109(60.9 \%)$ \\
\hline Black & $11(6.1 \%)$ \\
\hline $\begin{array}{l}\text { Other (American Indian/AK Native, } \\
\text { Asian/Pacific Islander) }\end{array}$ & $59(33.0 \%)$ \\
\hline \multicolumn{2}{|l|}{ Pathological differentiation } \\
\hline Poorly & $56(60.2 \%)$ \\
\hline Undifferentiated & 37 (39.8\%) \\
\hline Unknown & 86 \\
\hline \multicolumn{2}{|l|}{ Summary stage } \\
\hline Distant & $20(12.5 \%)$ \\
\hline Regional & $77(48.1 \%)$ \\
\hline Localized & $63(39.4 \%)$ \\
\hline Unstaged & 19 \\
\hline \multicolumn{2}{|l|}{ Primary site } \\
\hline Parotid gland & $153(85.5 \%)$ \\
\hline Submandibular gland & $23(12.8 \%)$ \\
\hline Major salivary gland, NOS* & $3(1.7 \%)$ \\
\hline \multicolumn{2}{|l|}{ Laterality } \\
\hline Left & $80(45.5 \%)$ \\
\hline Right & $96(54.5 \%)$ \\
\hline Unknown & 3 \\
\hline \multicolumn{2}{|l|}{ Tumor size } \\
\hline $\mathrm{T} 1$ & $17(21.3 \%)$ \\
\hline T2 & $29(36.2 \%)$ \\
\hline T3 & $21(26.3 \%)$ \\
\hline $\mathrm{T} 4$ & $13(16.2 \%)$ \\
\hline Unknown & 99 \\
\hline \multicolumn{2}{|l|}{ Lymph node metastases } \\
\hline NO & $42(46.7 \%)$ \\
\hline N1 & $47(53.3 \%)$ \\
\hline Unknown & 90 \\
\hline
\end{tabular}

Table 1 (continued)
Table 1 (continued)

\begin{tabular}{|c|c|}
\hline Characteristics & Total \\
\hline \multicolumn{2}{|c|}{ Distant metastases } \\
\hline MO & 87 (97.8\%) \\
\hline M1 & $2(2.2 \%)$ \\
\hline Unknown & 90 \\
\hline \multicolumn{2}{|l|}{ TNM stage } \\
\hline 1 & $14(17.1 \%)$ \\
\hline$\|$ & 16 (19.5\%) \\
\hline III & $21(25.6 \%)$ \\
\hline IV & $31(37.8 \%)$ \\
\hline Unknown & 97 \\
\hline \multicolumn{2}{|l|}{ Surgery } \\
\hline Yes & $166(92.7 \%)$ \\
\hline No & $13(7.3 \%)$ \\
\hline \multicolumn{2}{|l|}{ Radiotherapy } \\
\hline Yes & 127 (70.9\%) \\
\hline No & 52 (29.1\%) \\
\hline \multicolumn{2}{|l|}{ Chemotherapy } \\
\hline Yes & $33(18.4 \%)$ \\
\hline No/unknown & $146(81.6 \%)$ \\
\hline
\end{tabular}

*NOS: not stated. The type of major salivary gland of these 3 patients were not stated. LEC, Iymphoepithelial carcinoma; TNM, tumor-node-metastases.

node metastases $(\mathrm{P}<0.01$, Figure $2 B, C, D)$. Only 2 patients with distant metastases could survive more than 25 months. Divorced or widowed patient had worse prognosis than married or unmarried patients $(\mathrm{P}<0.01)$. In addition, the results showed no significant association of other characteristics and prognosis including gender $(\mathrm{P}=0.22)$, laterality $(\mathrm{P}=0.29)$, pathological grade $(\mathrm{P}=0.60)$, primary site $(\mathrm{P}=0.12)$.

\section{Effect of treatment regimen on prognosis}

Figure 3 showed the effect of different treatment regimens on prognosis. Patients received surgical resection had much longer overall survival time than those who did not received surgery (mOS: 219 vs. 68 months, $\mathrm{P}<0.01$ ). The use of radiotherapy could also prolong the overall survival of LEC-SG patients, but without significantly statistical difference (mOS: 231 vs. 138 months, $\mathrm{P}=0.11$; Figure 3B). 

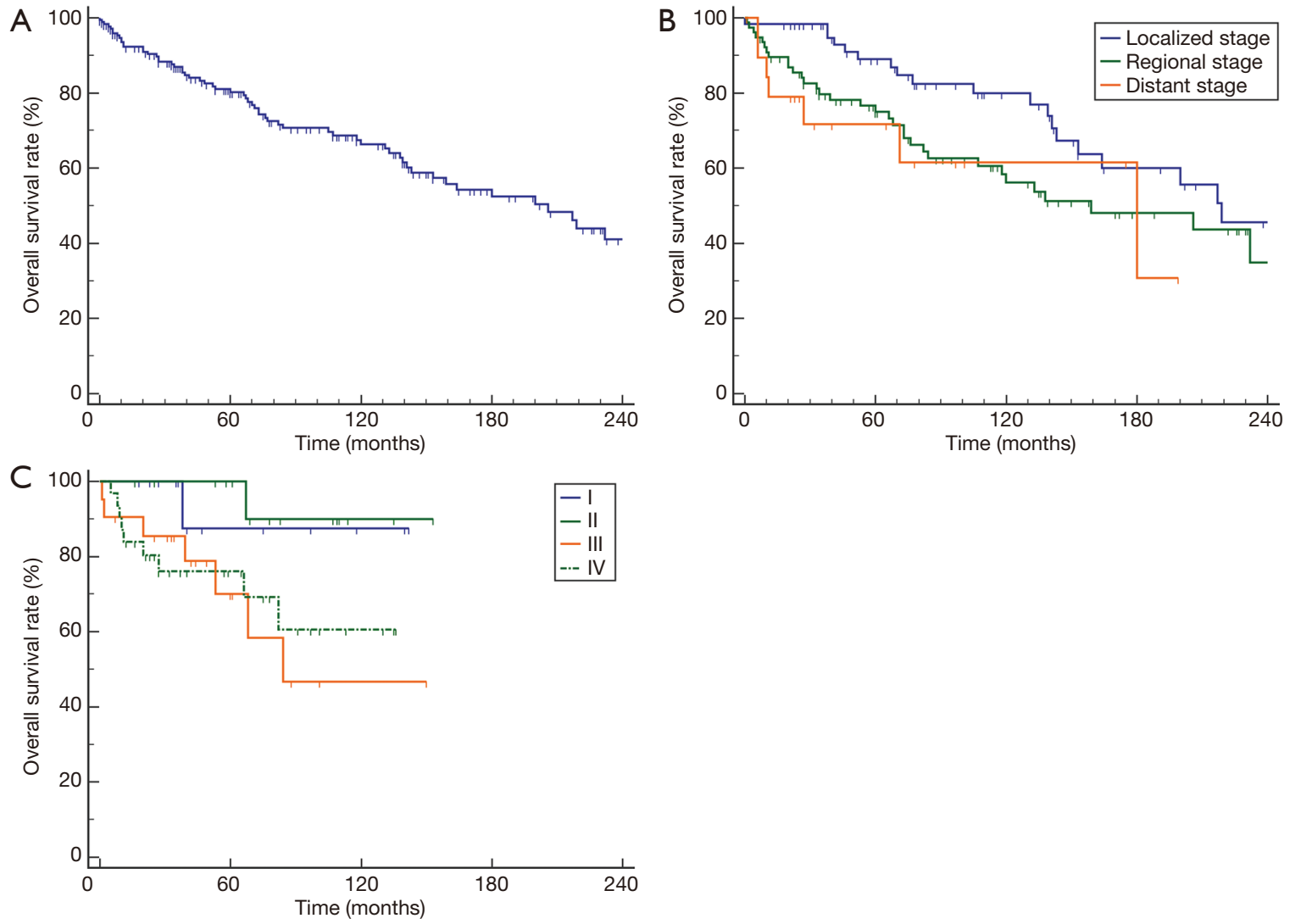

Figure 1 Overall survival of patients with LEC of salivary gland: (A) overall survival of all 179 patients; (B) overall survival stratified by SEER historic stage; (C) overall survival stratified by TNM stage. LEC, lymphoepithelial carcinoma; SEER, the Surveillance, Epidemiology, and End Results Database.

Meanwhile, there was no statistically significant difference in overall survival between LEC-SG patients with and without chemotherapy $(\mathrm{P}=0.18$; Figure $3 C)$. In addition, the combination of surgery and radiotherapy also could prolong the overall survival than those with surgery alone. Radiotherapy after surgery could improve long-term survival and decrease the risk of death among patients who survive exceed 10 year after surgery (232 vs. 143 months, $\mathrm{P}=0.25$; Figure $3 D$ ).

\section{Cox regression survival analyses}

The potential features affecting survival were evaluated using univariate and multivariate Cox regression model (Table 2). The univariate analysis revealed that age, race, marital status, lymph node metastases as well as the use of surgery were significantly associated with overall survival. Old age, non-white/black race, divorced/widowed status, lymph node metastases was associated with poor survival, while surgical resection was associated with good prognosis $(\mathrm{P}<0.05)$. The multivariate analysis showed that old age ( $>60$ years) as well as lymph node metastases were independent prognostic risk factors for LEC-SG patients. Conversely, the use of surgery was an independent favorable prognostic factor (HR =0.29, 95\% CI: 0.13-0.66; Table 2).

\section{Discussion}

The purpose of the present study was to elucidate the clinicopathological characteristics and prognosis of LECSG patients using the data of 179 patients from SEER database. The results showed that LEC of salivary gland had good prognosis with a mOS of 206 months, and old age, lymph node metastases as well as the use of surgery were independent prognostic factors.

LEC of salivary gland is exceedingly rare, it only 

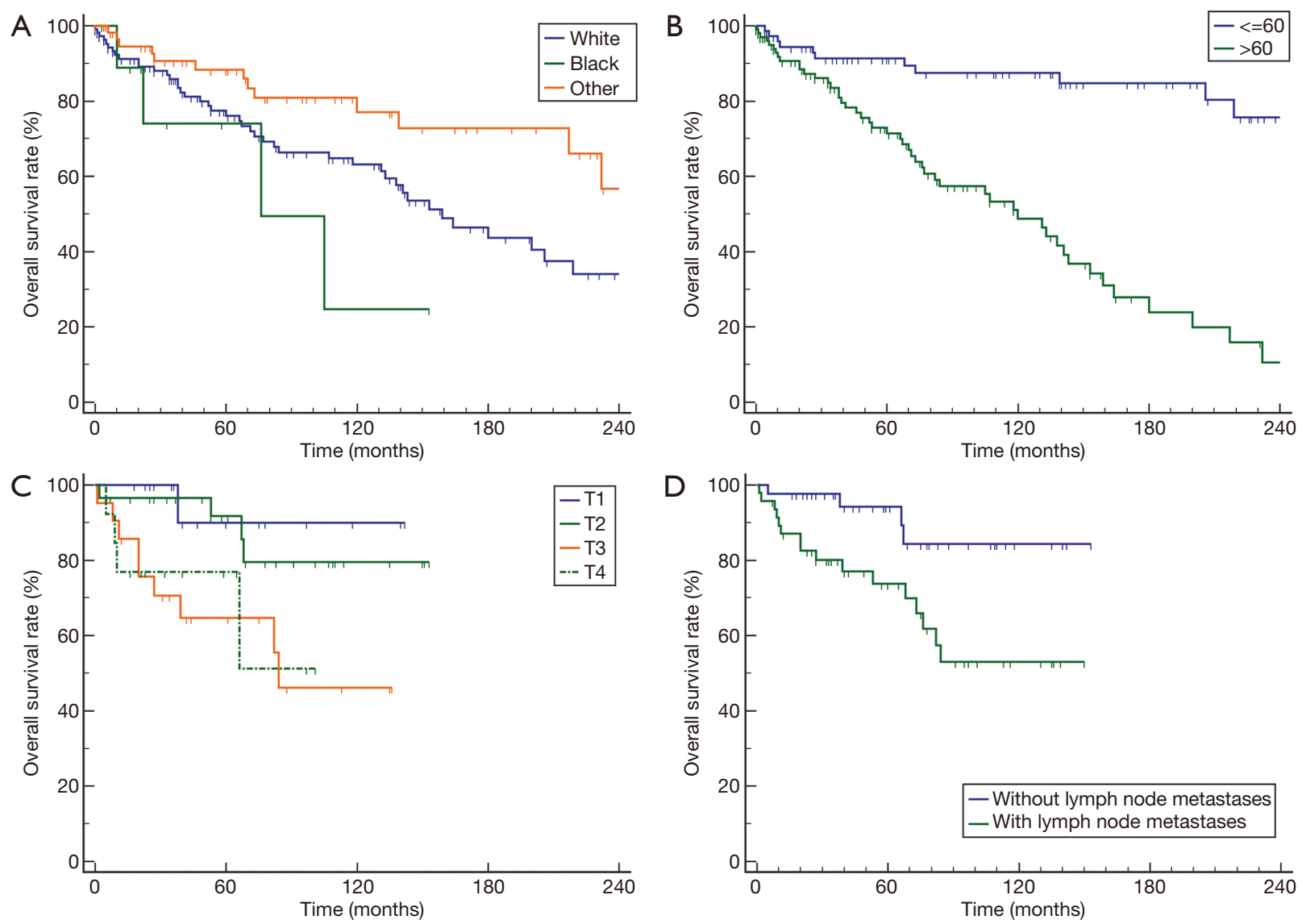

Figure 2 Overall survival for patients with salivary gland stratified by clinicopathological characteristics: (A) race; (B) age; (C) tumor stage; (D) lymph node metastases.

comprises $0.4 \%$ of salivary malignancies in Western country. Chinese patients have a relatively higher incidence of LEC, accounting for approximately $5-9.7 \%$ of all salivary gland tumors $(11,12)$. The present study showed that LEC of salivary gland is much more frequent in the non-white/black population than white or black population. Due to the invariable relationship between EBV and LEC, this regional difference in the occurrence of this disease may be attributed to cancer-related virus infection $(1,7)$. For example, several previous studies have reported a close correlation of EBV infection with LEC development in Southeast Asia, Greenland, and Alaska $(8,13,14)$. EBV positivity was also observed in LEC-SG patients from high incidence regions. For example, Ma found that EBV-DNA was positive in $43.9 \%(18 / 41)$ of Chinese LEC patients at initial diagnosis (8). The recent data demonstrated that $85.0 \%$ of lesions were located in parotid gland, which is in consistent with previous data $(15,16)$. Previous studies have some controversial results about the gender distribution of this disease. Wang YL, et al. reported a predominance of men with a female-to-male ratio of 1:1.75 among 11 cases with LEC of salivary gland (12). Another study including 103 cases reported this disease predominantly occurs in women, with a male-female ratio of 1:1.94 (16). Similar to Ma H's study, we also observed that $52 \%$ patients were women, with a female-to-male ratio of 1.08:1 (8). Several studies in Chinese population reported that the average age of LEC of salivary gland was around 50 years $(49.4-51.8$ years) $(8,11,16)$, but the age of patients in the present cohort was older than previous studies that the LEC-SG patients had an average age of 60.2 years, with 102 cases older than 60 years.

Among LEC-SG patients, several studies have reported nonspecific symptoms could lead to a delay in a definitive diagnosis of this malignancy, with a median delay period of 12 months (7 days to 20 years) $(8,11,16)$. Consequently, a majority of patients with LEC of salivary gland present 

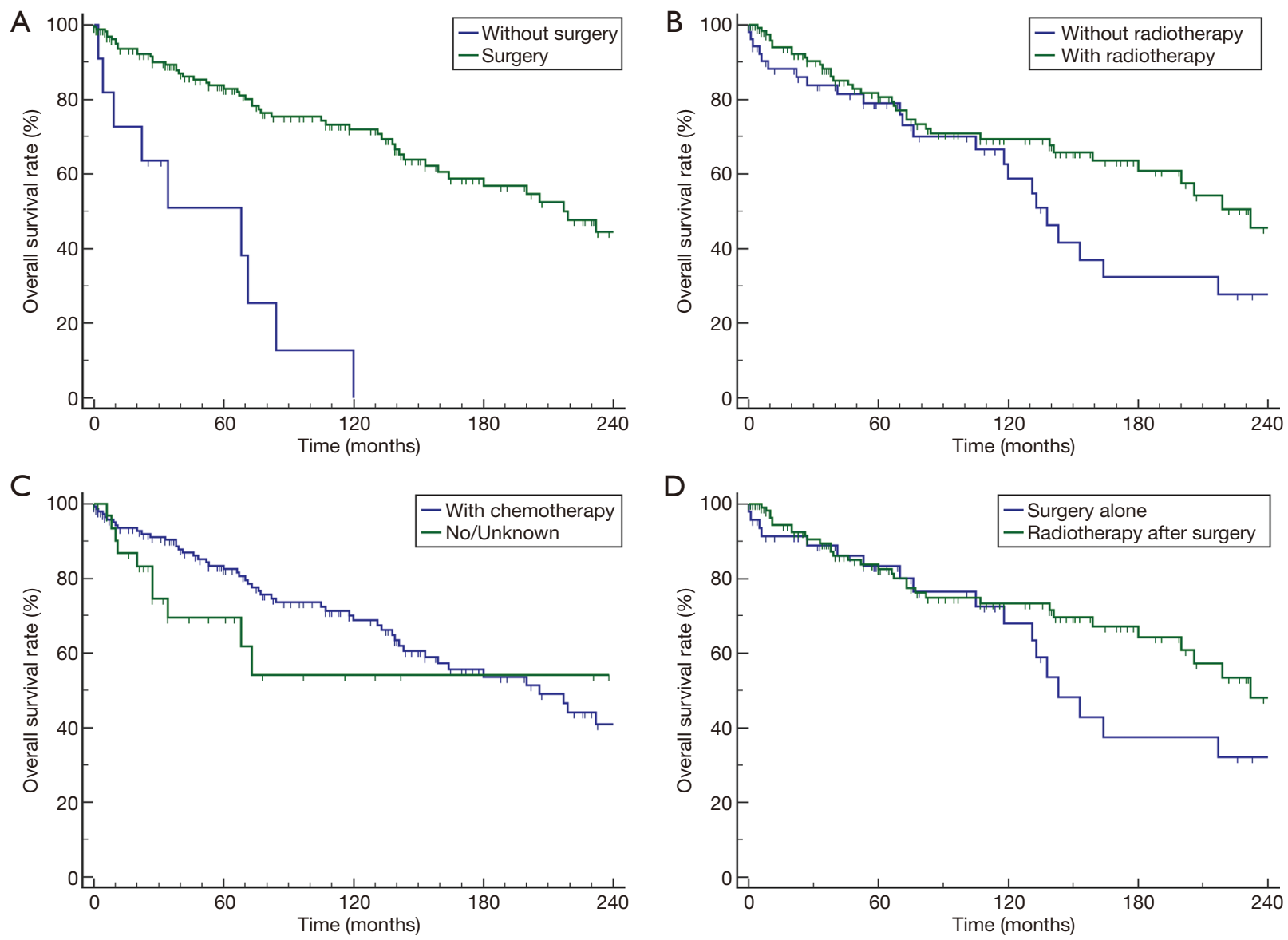

Figure 3 The effect of different treatments on overall survival for patients LEC of salivary gland: (A) surgery; (B) radiotherapy; (C) chemotherapy; (D) radiotherapy after surgery. LEC, lymphoepithelial carcinoma.

locally or regionally advanced disease at diagnosis. In $\mathrm{Ma}$ H' study, 59.4\% of patients were categorized into advanced stage (III/IV), and $43.5 \%$ of patients had lymph node metastases. In our study, the data revealed that $58.9 \%$ of patients were categorized into regional or distant stage at initial diagnosis, and $52.8 \%$ of patients had lymph node metastases. Only 2 cases in the present cohort had distant organ metastases, one case had bone metastases, the other one had no information about distant metastases.

In consistent with previous studies, the recent data also demonstrated that LEC-SG patients had a relatively better prognosis. The mOS for all LEC-SG patients was 206.0 months, with a 10 -year survival rate of $66.4 \%$. And, almost $38 \%$ of patients with LEC of salivary gland could survive exceed 20 years. The survival time of those patients is much longer than the reported data of other types of salivary gland malignancies including salivary malignant pleomorphic adenoma, mucoepidermoid carcinoma, adenoid cystic carcinoma $(8,11,16)$. As for treatment regimen, surgery is undoubtedly the preferred choice of treatment among patients with LEC of salivary gland. Even patients were diagnosed with stage IV, previous study reported $84.2 \%$ cases could receive neck dissection $(8,11,16)$. Our data showed that $92.7 \%$ of all patients and 29 of 31 stage IV patients had undergone surgery. Radiotherapy was also an important treatment regimen. In the present study, $70.9 \%$ patients received radiotherapy and $66.5 \%$ patients received postoperative radiotherapy. However, the survival analysis in the present cohort demonstrated that postoperative radiotherapy could not significantly prolong survival time when compared with surgery alone, but postoperative radiotherapy could improve the long-term survival. For example, the 10-, 15-year survival rates were $71.5 \%$ and $68.0 \%, 64.3 \%$ and $32.1 \%$ for patients who received postoperative radiotherapy or not, respectively. One possibility is that postoperative 
Table 2 Univariate and multivariate Cox proportional hazard analyses of the characteristics for overall survival in patients with LEC of salivary gland

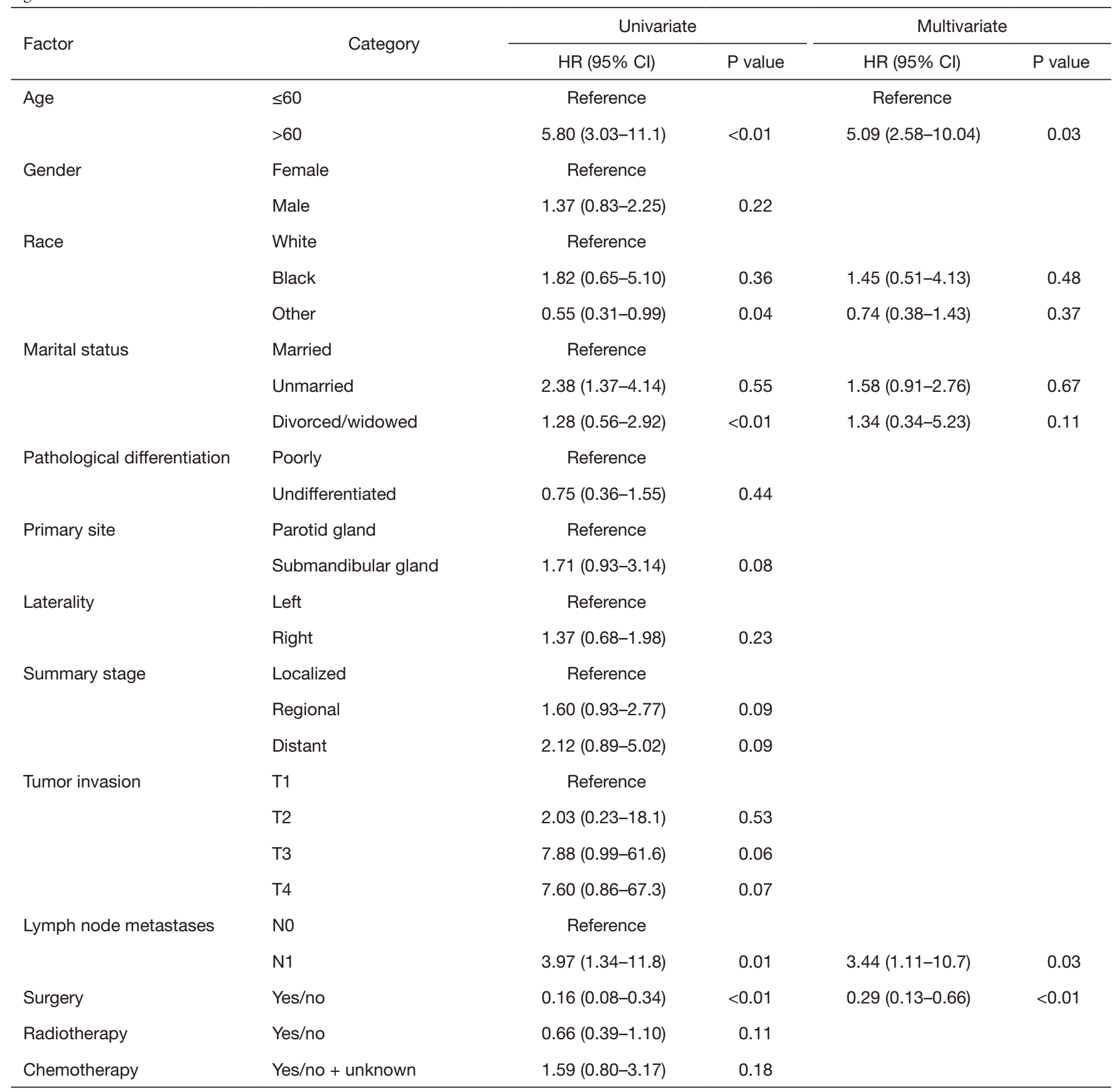

The COX analysis of distant metastases could not be conducted due to inadequate data. LEC, lymphoepithelial carcinoma; HR, hazard ratio; $\mathrm{Cl}$, confidence interval.

radiotherapy could decrease the risk of recurrence among those patients. For example, Ma H's study reported that postoperative radiotherapy was associated independently with relapse-free survival, but not overall survival $(8,11,16)$.
Therefore, postoperative radiotherapy was recommended for the patients with high-risk indications such as R1 resection with microscopically positive margins, lymph node metastases, etc. (17). Except for surgery and radiotherapy, 
chemotherapy could be also used in treatment of LECSG patients. In the present study, we could not observe any significant relationship of the use of chemotherapy with the overall survival among LEC-SG patients. As we known, no adequate information about the use of chemotherapy including chemotherapy regimens could be extracted from the SEER database, which limited the accuracy and reliability of the conclusion concerning the effect of chemotherapy among LEC-SG patients. For example, there are some similarities in histology between LEC and nasopharyngeal carcinoma, whether the commonly used chemotherapy regimens for nasopharyngeal carcinoma could also provide clinical benefit for LEC-SG patients still need to be further proven.

In conclusion, the results revealed that LEC-SG patients had a favorable prognosis and old age, lymph node metastases as well as the use of surgery were significantly associated with prognosis. Although there are several limitations similar to other analysis based on the SEER database including inadequate information, retrospective nature, small sample size, the present study is the largest series of cases with LEC-SG and could help the physician more deeply understand this malignancy.

\section{Acknowledgments}

Funding: None.

\section{Footnote}

Reporting Checklist: The authors have completed the STROBE reporting checklist. Available at http://dx.doi. org/10.21037/gs-20-464

Conflicts of Interest: All authors have completed the ICMJE uniform disclosure form (available at http://dx.doi. org/10.21037/gs-20-464). The authors have no conflicts of interest to declare.

Ethical Statement: The authors are accountable for all aspects of the work in ensuring that questions related to the accuracy or integrity of any part of the work are appropriately investigated and resolved. The study was conducted in accordance with the Declaration of Helsinki (as revised in 2013).

Open Access Statement: This is an Open Access article distributed in accordance with the Creative Commons
Attribution-NonCommercial-NoDerivs 4.0 International License (CC BY-NC-ND 4.0), which permits the noncommercial replication and distribution of the article with the strict proviso that no changes or edits are made and the original work is properly cited (including links to both the formal publication through the relevant DOI and the license). See: https://creativecommons.org/licenses/by-nc-nd/4.0/.

\section{References}

1. Wenig BM. Lymphoepithelial-like carcinomas of the head and neck. Semin Diagn Pathol 2015;32:74-86.

2. Coskun BU, Cinar U, Sener BM, et al. Lymphoepithelial carcinoma of the larynx. Auris Nasus Larynx 2005;32:189-93.

3. Kim YJ, Hong HS, Jeong SH, et al. Lymphoepithelial carcinoma of the salivary glands. Medicine (Baltimore) 2017;96:e6115.

4. Zhan KY, Nicolli EA, Khaja SF, et al. Lymphoepithelial carcinoma of the major salivary glands: Predictors of survival in a non-endemic region. Oral Oncol 2016;52:24-9.

5. Acuña G, Goma M, Temprana-Salvador J, et al. Human papillomavirus in laryngeal and hypopharyngeal lymphoepithelial carcinoma. Mod Pathol 2019;32:621-6.

6. Singhi AD, Stelow EB, Mills SE, et al. Lymphoepitheliallike carcinoma of the oropharynx: a morphologic variant of HPV-related head and neck carcinoma. Am J Surg Pathol 2010;34:800-5.

7. Cardesa A, Nadal A. Carcinoma of the head and neck in the HPV era. Acta Dermatovenerol Alp Pannonica Adriat 2011;20:161-73.

8. Ma H, Lin Y, Wang L, et al. Primary lymphoepitheliomalike carcinoma of salivary gland: sixty-nine cases with longterm follow-up. Head Neck 2014;36:1305-12.

9. Zhao W, Deng N, Gao X, et al. Primary lymphoepithelioma-like carcinoma of salivary glands: a clinicopathological study of 21 cases. Int J Clin Exp Pathol 2014;7:7951-6.

10. Schneider M, Rizzardi C. Lymphoepithelial carcinoma of the parotid glands and its relationship with benign lymphoepithelial lesions. Arch Pathol Lab Med 2008;132:278-82.

11. Tian Z, Li L, Wang L, et al. Salivary gland neoplasms in oral and maxillofacial regions: a 23 -year retrospective study of 6982 cases in an eastern Chinese population. Int J Oral Maxillofac Surg 2010;39:235-42.

12. Wang YL, Zhu YX, Chen TZ, et al. Clinicopathologic study of 1176 salivary gland tumors in a Chinese 
population: experience of one cancer center 1997-2007. Acta Otolaryngol 2012;132:879-86.

13. Hamilton-Dutoit SJ, Therkildsen MH, Neilsen NH, et al. Undifferentiated carcinoma of the salivary gland in Greenlandic Eskimos: demonstration of Epstein-Barr virus DNA by in situ nucleic acid hybridization. Hum Pathol 1991;22:811-5.

14. Saku T, Cheng J, Jen KY, et al. Epstein-Barr virus infected lymphoepithelial carcinomas of the salivary gland in the Russia-Asia area: a clinicopathologic study of 160 cases.

Cite this article as: Wang JQ, Deng RX, Liu H, Luo Y, Yang ZC. Clinicopathological characteristics and prognostic analysis of lymphoepithelial carcinoma of salivary gland: a populationbased study. Gland Surg 2020;9(6):1989-1997. doi: 10.21037/gs20-464
Arkh Patol 2003;65:35-9.

15. Ma S, Guan XY, Lee TK, et al. Clinicopathological significance of missing in metastasis B expression in hepatocellular carcinoma. Hum Pathol 2007;38:1201-6.

16. Wang P, Yang J, Yu Q. Lymphoepithelial carcinoma of salivary glands: CT and MR imaging findings. Dentomaxillofac Radiol 2017;46:20170053.

17. Agulnik M, McGann CF, Mittal BB, et al. Management of salivary gland malignancies: current and developing therapies. Oncol Rev 2008;2:86-94. 